\title{
Introducción a los mecanismos sociales y los consumos culturales en la sociología analítica
}

\author{
María Florencia Ares y Javier Maximiliano Paesani \\ Universidad Nacional de Salta - Sede Regional Tartagal \\ floren flo@hotmail.com
}

\begin{abstract}
Resumen
En este artículo presentamos y analizamos, en una dimensión epistemológica, una familia de explicaciones sociológicas enmarcadas en el enfoque general de lo que se ha denominado "sociología analítica", unificadas por una analogía básica: el mecanismo. La argumentación principal sigue ceñidamente a Hedström \& Bearman (2009). Se incluyen algunas aplicaciones presentadas a modo de ejemplo a los fines de aclarar el sentido de la propuesta mecanística. Finalmente, se señalan algunos núcleos problemáticos referidos al enfoque.
\end{abstract}

Palabras claves: mecanismo; modelo; acción; mercado cultural

\section{Resumo}

No presente artigo, apresentamos e analisamos, em uma dimensão epistemológica, uma família de explicações sociológicas enquadradas na abordagem geral do que tem sido chamado de "sociologia analítica" e unificadas por uma analogia básica: o mecanismo. O principal argumento segue de perto Hedström \& Bearman (2009). Algumas aplicações apresentadas como exemplos estão incluídas para esclarecer o significado da proposta mecanicista. Por fim, alguns núcleos problemáticos referentes à abordagem são apontados.

Palavras chaves: mecanismo; modelo; ação; mercado cultural

\section{Introducción}

En la comprensión y explicación de los fenómenos del universo, la mente humana hace uso de metáforas y analogías (Lakoff \& Johnson: 1980, Hofstadter \& Sander: 2013). También es posible detectarlas en el discurso científico, y ello ha merecido un creciente interés por parte de los epistemólogos. Es justo mencionar, además, que el fenómeno ya era conocido por los 


\section{REVCOM \\ REVISTA CIENTIFICA DE LA REDCOM}

REVCOM | ISSN 2451-7836 | Año 4, \#8 | mayo de 2019 | Contribuciones | e011

historiadores de la ciencia (véase, por ejemplo: Kuhn: 1996, y Westfall: 1980). En el presente trabajo, nos ocupamos de presentar y explorar sumariamente una analogía, la del mecanismo, en un ámbito científico particular: la explicación analítica de los fenómenos sociales. Exploramos, además, algunas aplicaciones específicas, como el estudio de la segregación social y, más relevante a la problemática de la comunicación, el estudio de cierto tipo de influencia social en el consumo de bienes culturales.

\section{Analogías científicas}

La revolución científica moderna se desarrolla al filo de la concepción mecanicista del universo y la analogía de los mecanismos (Westfall: 1980). Si bien en el ámbito de las ciencias naturales el mecanicismo perdió fuerza a fines del siglo XIX y comienzos del XX, en particular con los desarrollos de las teorías estadísticas de los procesos físicos, en la explicación de la conducta social una cantidad creciente de autores lo recuperan. En este sentido, podríamos decir que llegan con algo de retraso. Se verifica una tendencia al cambio de analogía hacia lo informático-computacional, incluso en las teorías fundamentales de los procesos materiales (en lo personal, nos parecen especulaciones prematuras, pero en ciencia es difícil establecer por dónde puede venir la siguiente revolución). En el ámbito de la explicación de la conducta humana, la teoría computacional de la inteligencia puede considerarse vanguardista, en este sentido, en tanto explicación de la conducta humana y los procesos psicológicos superiores (que son parte fundamental de la concepción teórica que comentaremos), en términos de sistema informacionales y computacionales. La analogía del mecanismo, y sus modelos asociados, podrían operar como punto de inflexión hacia el desarrollo de lo que, con sobrio entusiasmo, ha empezado a denominarse "ciencia social computacional" (Watts: 2013).

Los métodos analíticos en ciencias sociales exploran mecanismos generadores de hechos sociales. Estos mecanismos se refieren a acciones de los individuos y a relaciones que los vinculan. La postura resultante puede denominarse "individualismo estructural". Es una doctrina metodológica según la cual los hechos sociales deben explicarse como resultados, intencionales o no, de las acciones individuales. El individualismo estructural difiere del individualismo metodológico tradicional al atribuir una importancia explicativa sustancial a las estructuras sociales en las que los individuos están insertos. 


\section{VREVCOm \\ REVISTA CIENTIFICA DE LA REDCOM}

REVCOM | ISSN 2451-7836 | Año 4, \#8 | mayo de 2019 | Contribuciones | e011

\section{Explicaciones mecanísticas}

No existe un consenso sobre la definición de "mecanismo" en Sociología. Según los autores a considerar encontramos diferencias y matices. Pero todas las definiciones subrayan la necesidad de hacer inteligibles las regularidades mediante la observación de las entidades y dinámicas que las hacen posibles. (Hedström \& Bearman, 2009; Elster: 1999, 2007; Bunge: 2007)

Considérese, por ejemplo, la siguiente cita:

Las explicaciones mecanísticas tratan a los sistemas productores de un comportamiento determinado de una manera análoga a las máquinas ... Una máquina es un compuesto de partes interrelacionadas ... que se combinan de tal forma que cada una contribuye a producir el comportamiento del sistema. Una explicación mecanística identifica estas partes y su organización, mostrando cómo el comportamiento de la máquina es una consecuencia de las partes y su organización. (Bechtel y Richardson: 1993: 17, citado en Hedström \& Bearman: 2009: 5).

Hedström y Bearman (2009) recomiendan no tomarse muy en serio la metáfora de las máquinas. Sin resaltar los aspectos más físicos y literales, la idea central del enfoque es que se explica identificando partes componentes cuya dinámica conjunta produce los hechos sociales observados.

Machamer, Darden \& Craver (2000) sostienen que un mecanismo consiste de entidades (con sus propiedades) y las actividades que realizan estas entidades, ya sea por sí mismas o en concierto con otras entidades. Estas actividades provocan cambios. El tipo de cambios producido es función de las propiedades y actividades de las entidades, como de las relaciones que se establecen entre ellas. Un mecanismo, así definido, se refiere a una constelación de entidades y actividades que están organizadas de manera tal que producen regularmente un tipo particular de resultado. Explicamos un resultado observado refiriendo al mecanismo que lo produce regularmente.

No existe oposición estricta entre los análisis cuantitativos de la sociología tradicional y la explicación mecanística. En realidad, el análisis cuantitativo puede resultar indispensable para discriminar entre mecanismos explicativos rivales; o para distinguir, en mecanismos postulados, entre actividades y relaciones relevantes e irrelevantes. A su vez, como ha sido señalado a 
REVCOM | ISSN 2451-7836 | Año 4, \#8 | mayo de 2019 | Contribuciones | e011

menudo, una correlación estadística puede adquirir fuerza explicativa al ser reforzada mediante un mecanismo que explique cómo se produce la relación entre los datos.

Los sociólogos analíticos suelen coincidir en la necesidad de construir y afinar una caja de herramientas compuesta de mecanismos generativos semigenerales, enmarcándose en la tradición mertoniana, que enfatiza en las teorías explicativas de rango medio con un fuerte escepticismo respecto a las teorías muy generales. Y en algunos casos (Elster; 2007, 2012) con un escepticismo sólidamente fundamentado respecto a las ambiciones excesivas de ciertos enfoques en ciencias sociales, cuestión discutida en las conclusiones.

\section{Individualismo estructural}

Las sociologías analíticas, con los mecanismos que conjeturan, pueden modular diversamente la cuestión del individualismo metodológico. Según argumenta Elster (2007:36): "En las ciencias sociales, una explicación satisfactoria debe estar anclada últimamente en hipótesis sobre la conducta individual". Serían, según esto, la Psicología y eventualmente la Biología, las ciencias que deberían ofrecer los fundamentos de nivel básico para construir las explicaciones sobre hechos sociales. El argumento adquiere fuerza en la medida en que se pretende entroncar a las ciencias sociales en el conjunto más amplio del conocimiento científico en general. De otro costal es el problema de la existencia de suficiente desarrollo en las ciencias de base para proceder a las reducciones necesarias (Elster en particular es escéptico respecto a las explicaciones naturalistas en el estado actual de la Biología).

Dejando a un lado las cuestiones ontológicas y epistemológicas generales relativas a la unidad de la ciencia, pueden establecerse argumentos metodológicos ulteriores en favor del enfoque individualista. Por ejemplo:

Los débiles efectos agregados de la violencia televisiva sobre la violencia en la vida real podrían enmascarar fuertes efectos opuestos sobre determinados subgrupos. En todos estos casos, un agregado neutral podría reflejar o bien una población homogénea de individuos no afectados o una población heterogénea de individuos fuertemente afectados, pero en direcciones opuestas. La necesidad de disipar esta ambigüedad proporciona un argumento más a favor del individualismo metodológico. Para explicar el comportamiento en el nivel agregado, debemos observar el comportamiento de los componentes individuales. (Elster: 2007: 42). 


\section{$\checkmark$ REVCOm \\ REVISTA CIENTIFICA DE LA REDCOM}

REVCOM | ISSN 2451-7836 | Año 4, \#8 | mayo de 2019 | Contribuciones | e011

El individualismo estructural, en cambio, es una doctrina metodológica según la cual todos los hechos sociales, su estructura y cambio, en principio, son explicables en términos de individuos, sus propiedades, acciones y relaciones entre sí. Difiere del individualismo fuerte al estilo de Elster al incorporar las relaciones y estructuras relacionales que configuran los agentes en sus acciones colectivas.

El individualismo estructural no implica, tampoco, un compromiso con ningún tipo específico de motivo o estado intencional que explicaría por qué los individuos actúan como lo hacen. Por lo tanto, no obliga a comprometerse con ninguna forma de teoría de la elección racional, y, en su mínima expresión, puede no hacer referencia a estados mentales o intencionales en absoluto. En el individualismo estructural pueden reconocerse, entre otros, dos aspectos importantes: la preocupación weberiana por la acción orientada a los demás y la preocupación del enfoque basado en mecanismo por la profundidad causal.

\section{Alcanzando profundidad causal}

La aspiración a profundidad causal quiere decir la identificación explícita de los microfundamentos, "engranajes y ruedas sociales" [cogs \& wheels], a través de los cuales se producen los hechos sociales a explicar. Es decir, las acciones y relaciones. Las acciones son importantes porque a la Sociología le interesan, entre otras cuestiones, los resultados, intencionales o no, de las acciones de los individuos. Las acciones individuales típicas están orientadas hacia otros, y, por lo tanto, las relaciones con otros deben tenerse en cuenta al explicar por qué los individuos hacen lo que hacen. Por otra parte, las relaciones sociales son centrales para explicar por qué, actuando como lo hacen, los individuos producen los resultados sociales que obtienen. Sin embargo, que las relaciones sean valiosas para explicar los resultados no significa que sean independientes de los individuos y sus acciones. Las estructuras relacionales son explicables como resultados, intencionales o no, de la acción individual.

Hedström \& Bearman (2009) señalan como ejemplo el caso de las cadenas de vacantes. Una jubilación, motivada quizás por el deseo de una vida más tranquila, crea una oportunidad para los demás, es decir, una vacante que espera ser ocupada por un nuevo ocupante. La vacante generada por la jubilación es ocupada por otra persona cuya razón para aceptar el trabajo, tal vez, es obtener más estatus, o un salario más alto, o simplemente un cambio de lugar, pero esto crea otra vacante en el trabajo anterior de esta persona, y de esta forma, las 
REVCOM | ISSN 2451-7836 | Año 4, \#8 | mayo de 2019 | Contribuciones | e011

cadenas de vacantes crean interdependencias sociales que son importantes para explicar la movilidad. Los deseos de las personas - de jubilación, promoción, o cambio de lugar- motivan al sistema. Sin tales orientaciones, las personas pueden no moverse. Pero los componentes esenciales no se encuentran únicamente en las intenciones de los actores. La profundidad causal se logra al reconocer que la acción tiene lugar en las estructuras sociales que en este caso canalizan las oportunidades de movilidad y, por lo tanto, explican por qué observamos lo que observamos.

\section{El vínculo micro-macro}

Las teorías pueden construirse partiendo de un estado de naturaleza idealizado en el cual no se asume que las relaciones sociales existan o que importen. Tales experimentos mentales pueden ser desafiantes y entretenidos, incluso útiles en la teoría orientada normativamente, pero no es probable que aporten demasiado a la hora de explicar fenómenos sociales reales. Es claro que muchos componentes esenciales de las explicaciones sociológicas, como las normas y las redes, a menudo son el resultado de procesos sociales largos e intrincados. Si buscamos explicaciones centradas exclusivamente en las acciones individuales, tales componentes deben, o bien ser ignorados, lo que parece inaceptable; o deben ser explicados, lo que, dado el estado actual de la teoría social, es en muchos casos imposible. Por esta razón, el realismo y la precisión de la explicación propuesta mejorarán enormemente si consideramos dadas ciertas propiedades de nivel macro, como las estructuras relacionales, y las incorporamos en la explicación. 


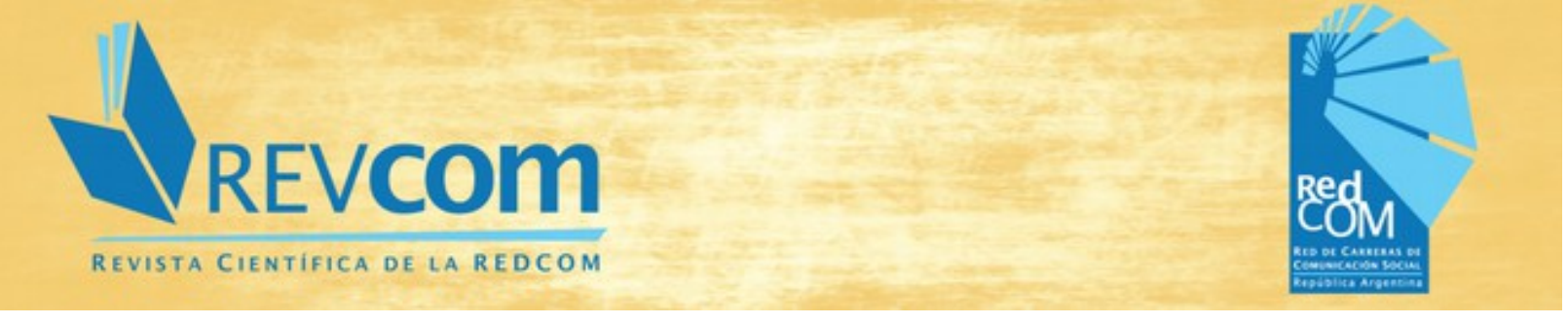

REVCOM | ISSN 2451-7836 | Año 4, \#8 | mayo de 2019 | Contribuciones | e011

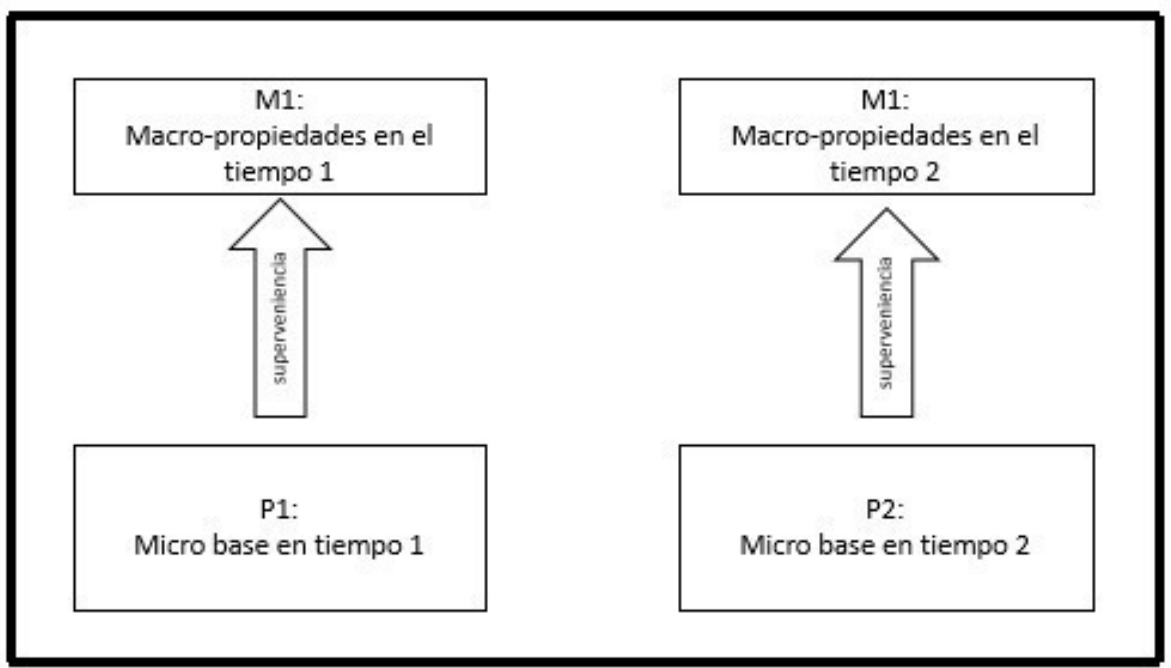

Fig. 1.1 Relaciones micro-macro como relaciones de superveniencia. Adaptado de Hedström \& Bearman 2009.

La sociología analítica busca esclarecer los vínculos entre lo micro y lo macro. Pero ¿qué es una macro-propiedad? Las propiedades macro son propiedades de una colectividad o un conjunto de entidades de nivel micro, pero que no son definibles para una única entidad de nivel micro. Ejemplos importantes de tales propiedades de nivel macro incluyen acciones típicas, creencias, deseos, etc. entre los miembros de la colectividad, así como distribuciones y patrones de agregación que caracterizan a la colectividad, como la desigualdad, la segregación espacial y las redes.

La relación entre niveles micro y macro es, en esta concepción, similar a lo que en el análisis filosófico del problema mente-cuerpo a veces se denomina 'superveniencia' (por ejemplo, Kim: 2011), y se representa en la Figura 1.1. Utilizando el lenguaje de la superveniencia, una propiedad macro, $\mathrm{M}$, sobreviene en un conjunto de propiedades de nivel micro, $\mathrm{P}$, si la identidad en $\mathrm{P}$ necesariamente implica identidad en $\mathrm{M}$. Si la propiedad macro es superveniente sobre el nivel micro, eso significa que, si dos colectividades o sociedades son idénticos entre sí en términos de sus propiedades de nivel micro, entonces sus propiedades de nivel macro también serán idénticas. También implica que dos colectividades que difieren en sus propiedades de nivel macro también difieren necesariamente en sus propiedades de nivel micro. Pero no implica que dos colectividades con una propiedad de nivel macro idéntica 
REVCOM | ISSN 2451-7836 | Año 4, \#8 | mayo de 2019 | Contribuciones | e011

necesariamente tengan propiedades de micronivel idénticas, porque las propiedades idénticas de nivel macro se pueden producir de diferentes maneras.

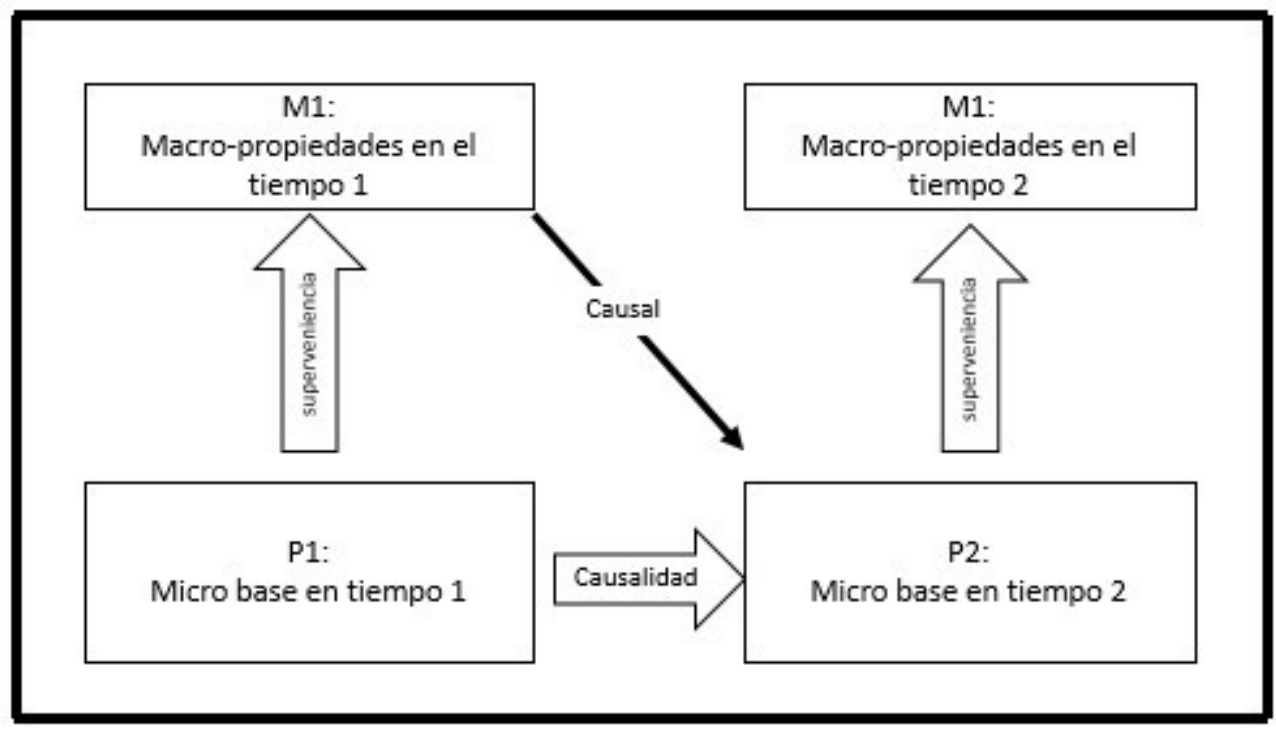

Fig. 1.2 Macrodinámica desde una perspectiva de superveniencia. Adaptado de Hedström \& Bearman 2009.

Aunque lo macro depende de las relaciones micro, micro a macro o $\mathrm{P}$ a $\mathrm{M}$ no deben verse como relaciones causales. Las propiedades macro siempre se instancian al mismo tiempo que las propiedades micro sobre las que sobrevienen, y un grupo o una sociedad tiene las propiedades macro que tiene en virtud de las propiedades y relaciones de sus entidades de nivel micro. La relación micro-macro es una relación de partes a un todo en lugar de una relación de causa a efecto. Por ejemplo, si existe un conjunto de relaciones diádicas entre los miembros de un grupo, estas relaciones diádicas no causan la estructura de red que vincula a los individuos entre sí: la constituyen. De manera similar, las propiedades de los individuos que residen en diferentes ubicaciones espaciales no causan la extensión de la segregación residencial: la constituyen.

Otra implicación importante de la perspectiva de superveniencia es que si una propiedad macro o micro en un período de tiempo anterior, $\mathrm{P} 1 \circ \mathrm{M} 1$, influye causalmente en una propiedad de nivel macro en el presente, M2, debe hacerlo al influir en su base de superveniencia, P2 (ver Fig. 1.2). Los ejemplos sociológicos de relaciones causales del tipo M1-a-P2 incluyen procesos que operan "a espalda" de individuos, como diversos procesos de socialización, pero las propiedades a nivel macro también pueden tener eficacia causal al ser 


\section{REVCOm \\ REVISTA CIENTIFICA DE LA REDCOM}

REVCOM | ISSN 2451-7836 | Año 4, \#8 | mayo de 2019 | Contribuciones | e011

componentes que los individuos toman conscientemente en cuenta al decidir qué hacer. Un ejemplo de esto es cuando la decisión de un individuo de unirse o no a un movimiento social está influenciada por el número de otras personas que ya se han unido al movimiento. Otros ejemplos de tales decisiones son las de si votar, si trasladarse a un nuevo vecindario, si comprar el último best-seller y si descargar o no una canción de un sitio web. Aunque se pueden observar regularidades sistemáticas en el nivel $\mathrm{M}$, tales asociaciones típicamente dicen poco sobre por qué observamos lo que observamos. Desde esta perspectiva, tales asociaciones son fenómenos superficiales que emergen de y requieren explicaciones en términos de procesos subyacentes más profundos. El punto importante es que, en cada instancia de tiempo, macro, $\mathrm{M}$, es superveniente en micro, $\mathrm{P}, \mathrm{y}$ en orden explicar los cambios en $\mathrm{M}$ debemos explicar los cambios en esta micro base. Se requeriría en muchos casos, según esto, de herramientas teóricas y empíricas para el análisis de sistemas complejos de acciones individuales relacionadas. Los sociólogos han chocado con límites en el pasado en este respecto, pero algunos investigadores se entusiasman con las posibilidades que abre el desarrollo explosivo de los sistemas de computación en décadas recientes (Watts: 2013). Por otra parte, si bien las dinámicas observadas pueden ser complejas, el enfoque confía en la capacidad generativa de los modelos simples para dar cuenta del resultado.

\section{Ejemplos de modelos simples}

Para ilustrar lo dicho hasta aquí, tomamos algunos ejemplos de modelos simples basados en explicaciones mecanísticas.

Se ha argumentado (véase, por ejemplo, Harford: 2008) que la extrema segregación racial que existe en algunas ciudades de Estados Unidos, puede no indicar la existencia de un profundo racismo. La segregación -sea por raza, por clase social o por nivel económico- puede ser el resultado de preferencias y prejuicios muy moderados. Thomas Schelling descubrió que "una muy pequeña preferencia por no tener como vecinos a demasiadas personas diferentes a ti, o incluso, sencillamente, la preferencia por tener algunas personas del vecindario que sean como tú...podría conducir a radicales efectos en el equilibrio que se asemejarían mucho a una segregación extrema". (Harford: 2008: 171)

En el modelo de segregación de Thomas Schelling (Schelling: 1971), tenemos dos tipos de individuos, a los que podemos referirnos como Blancos y Grises, y que se distribuyen en un reticulado. Cada individuo decide dónde vivir en el reticulado, pero la decisión está influenciada 
REVCOM | ISSN 2451-7836 | Año 4, \#8 | mayo de 2019 | Contribuciones | e011

por las decisiones pasadas de otros. Hay restricciones, sin embargo. Las personas solo pueden mudarse a puestos vacantes, y prefieren vivir cerca de algunos de su propia clase. Si los Blancos y los Grises se distribuyen aleatoriamente a través del espacio social representado por el reticulado, muchos de ellos estarán insatisfechos, ya que tendrán muy pocos vecinos de su propio tipo. Esto los motivará a mudarse a nuevas ubicaciones, pero al ingresar pueden inclinar el balance de la población local allí, haciendo que otros se vayan. Si unos pocos Grises se mudan a un área, los Blancos locales podrían irse, y cuando lleguen a sus nuevas áreas, podrían hacer que Grises se vayan, y así sucesivamente.

Con un tablero de ajedrez y un puñado de fichas blancas y negras puede instanciarse una versión elemental del modelo. Primero, se colocan las fichas de forma alterna sobre el tablero y se dejan vacíos los cuatro cuadrados de las esquinas. Las fichas negras y blancas representan dos tipos distintos de personas: blancos y negros. Cada uno de ellos tiene como máximo 8 vecinos y como mínimo, 4. Todos tienen una misma preocupación: no ser ampliamente superados en número por los otros en sus propios barrios. Aceptarían una leve mayoría del otro color, pero se mudarían al descubrir que los superan en más de dos tercios.

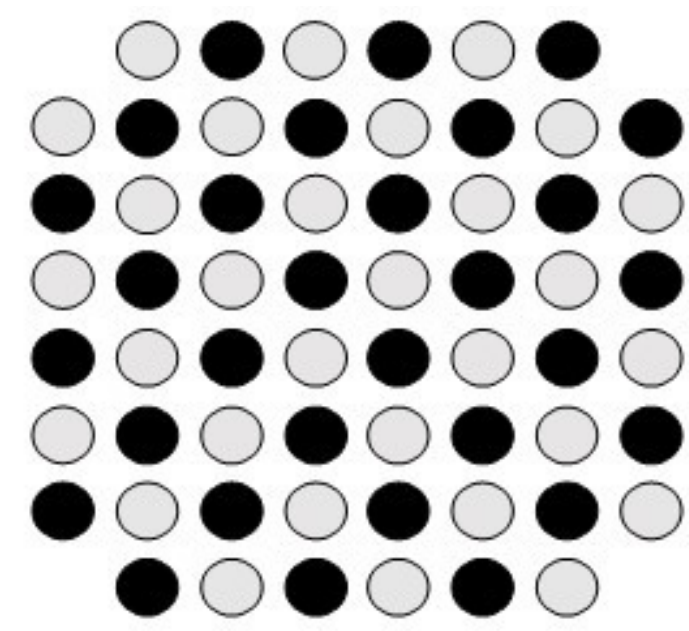

Fig. 2.1 Tablero de ajedrez de Tomas Schelling: una utopía perfectamente integrada. Adaptado de Harford (2008).

Se puede considerar a este tablero como un modelo de sociedad integrada. Blancos y negros viven uno al lado de otros. Sus preferencias son moderadas. Tienen vecinos de otro color, pero también tienen cerca vecinos de su mismo tipo. Sin embargo, es una sociedad muy 
REVCOM | ISSN 2451-7836 | Año 4, \#8 | mayo de 2019 | Contribuciones | e011

frágil. Para demostrarlo se quitan del tablero veinte fichas al azar y se añaden, también al azar, otras cinco. El resultado es el siguiente:

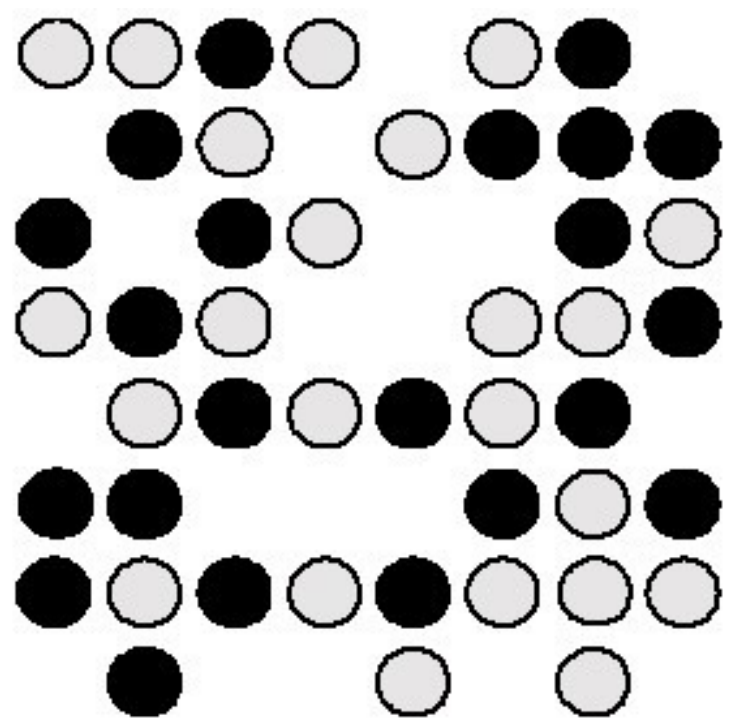

Fig. 2.2 El mismo tablero, después de quitar veinte piezas y añadir, al azar, otras cinco. Adaptado de Harford, 2008

En el nuevo tablero las fichas aparecen más desordenadas. Hay algunas fichas blancas y negras que tienen más del doble de vecinos de otro color. Si desplazáramos estas fichas a la posición más cercana donde no estuvieran tan ampliamente superadas en número por los de otro tipo, los desplazamientos producirían una reacción en cadena, generando segregación como resultado. Es decir, una concentración de las fichas de cada color en determinados lugares del tablero. 


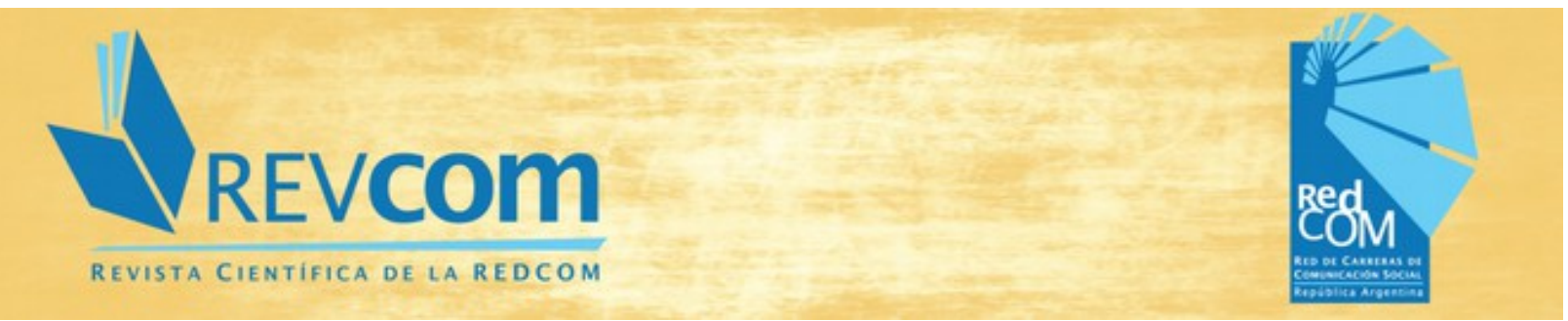

REVCOM | ISSN 2451-7836 | Año 4, \#8 | mayo de 2019 | Contribuciones | e011

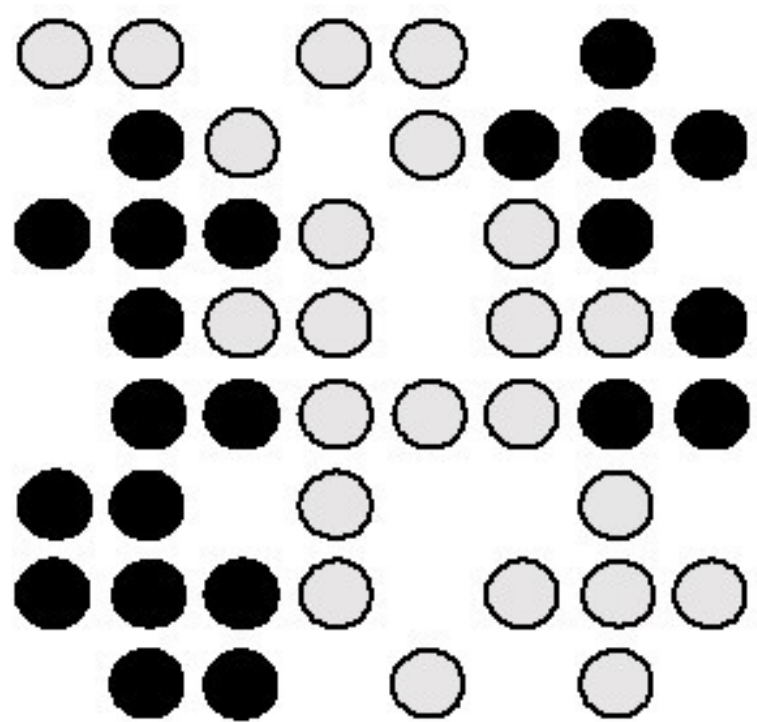

Fig. 2.3 Posición final, segregada, después de que las piezas hayan sido desplazadas a sus lugares de preferencia. Adaptado de Harford, 2008.

Schelling mostró que incluso si los individuos tienen preferencias fuertes por vecindarios mixtos, la segregación a menudo surge como una consecuencia involuntaria. Por ejemplo, si los blancos y los grises quieren al menos 26 por ciento de su propia clase en el vecindario para quedarse, producen un resultado colectivo donde el vecindario promedio consiste en más del 70 por ciento del mismo tipo (Harford, 2008). (Fig. 2.1)

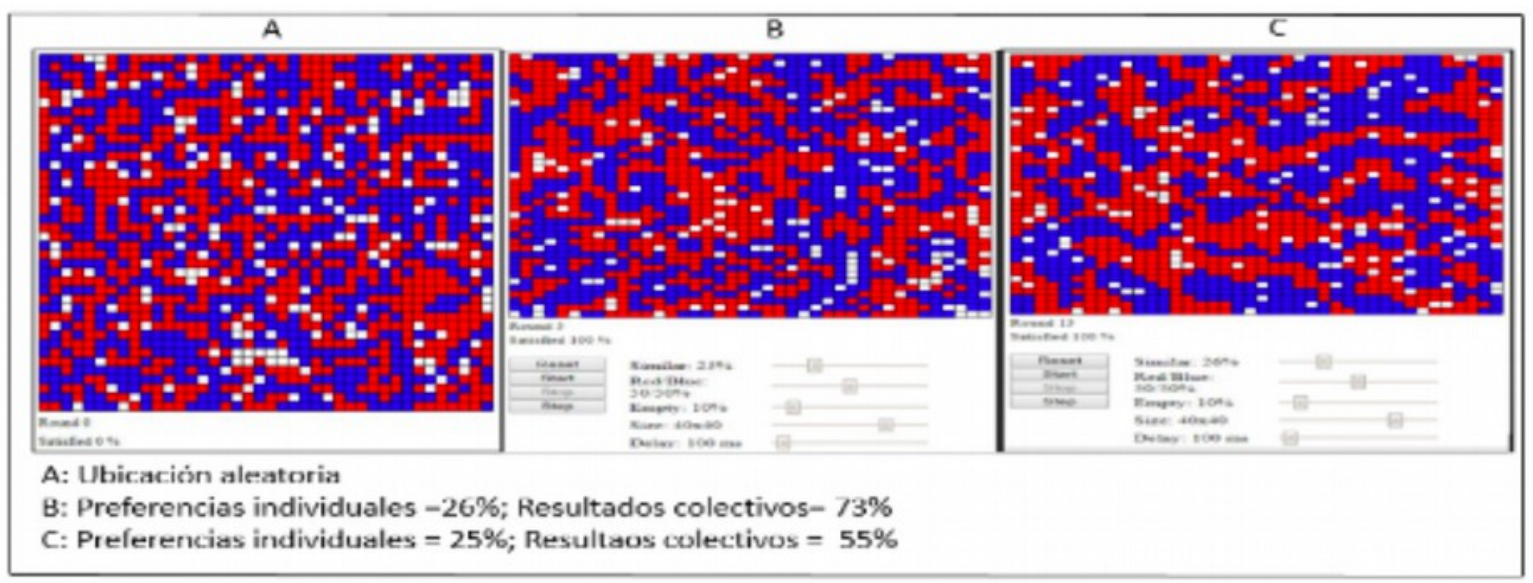

Fig.3.1 Simulación de preferencia individuales y segregación residencial. Disponible en: McCown,F. (2019).

Schelling's Models of Segregation. Computer Science Department

Harding University. http://nifty.stanford.edu/2014/mccown-schelling-model-segregation/

"Introducción a los mecanismos sociales y los consumos culturales en la sociología analítica" | María Florencia Ares y Javier Maximiliano Paesani | https://doi.org/10.24215/24517836e011 | Pág. 12 
REVCOM | ISSN 2451-7836 | Año 4, \#8 | mayo de 2019 | Contribuciones | e011

Schelling (1971) también mostró que diferencias pequeñas en el nivel micro, y en apariencia triviales, pueden hacer grandes diferencias en el nivel macro. Si los individuos que habitan la retícula desean al menos 25 en lugar del 26 por ciento de su propia clase en sus vecindarios, observaremos una macroestructura como la del Panel $C$, un patrón considerablemente menos segregado que el del Panel B.

Si observamos únicamente los resultados en el nivel macro, podríamos llegar a la conclusión de que la sociedad representada en el Panel B está compuesta por individuos con prejuicios más fuertes que los individuos que componen el Panel $\mathrm{C}$, tomando como premisa las diferencias en los patrones de segregación observados. Pero sería un error, puesto que las diferencias individuales en las preferencias son mínimas.

Éste es un resultado muy importante que se extiende mucho más allá de este ejemplo estilizado. Ha sido producido muchas veces en contextos diferentes; por ejemplo, en el trabajo de Granovetter (1978) sobre la acción colectiva. Lo que cabe destacar es que los resultados y las relaciones observados en el nivel macro no suelen explicar, según estas concepciones, las razones de su aparición. Infererir causalmente sobre la base de datos en el nivel macro puede conducir a errores.

Cabe realizar una advertencia ulterior. Si vemos estas simulaciones a la luz de la Figura 1.2, se observa que los resultados complejos e inesperados no tienen nada que ver con la complejidad del enlace micro-macro o con cualquier forma misteriosa de "emergencia". En cada instante del tiempo el vínculo de lo micro a lo macro es, simplemente, una relación de superveniencia. La complejidad emerge de la dinámica del conjunto de acciones individuales relevantes.

De esto se sigue un importante punto metodológico: para comprender la dinámica colectiva debemos estudiar la colectividad como un todo, pero no debemos estudiarla como una entidad con agencia independiente de las acciones de los individuos que relaciona. Solo al tomar en cuenta las entidades individuales, y de manera más crítica las relaciones entre ellas y sus actividades, podemos entender la estructura macro que observamos. 
REVCOM | ISSN 2451-7836 | Año 4, \#8 | mayo de 2019 | Contribuciones | e011

\section{Mecanismos de selección}

Se ha sugerido que los mecanismos de selección son centrales para explicar un rango de fenómenos diferentes. En su forma más básica es un tipo de mecanismo que vincula la propiedad de una entidad a su éxito reproductivo en un ambiente o entorno específico.

El esquema central para el mecanismo de selección se puede resumir de la siguiente manera:

- Un conjunto de entidades, $\boldsymbol{e}_{1}$ a $\mathbf{e}_{n}$, que varían con respecto a una propiedad, $\boldsymbol{p}_{i}$.

- En un cierto ambiente, el beneficio, $\boldsymbol{b}_{\boldsymbol{i}}$, otorgado a la entidad $\boldsymbol{i}$ es una función de su propiedad $\boldsymbol{p}_{\boldsymbol{i}}$.

- El valor de $\boldsymbol{b}_{\boldsymbol{i}}$ influye en la frecuencia relativa de entidades con diferentes propiedades en períodos de tiempo posteriores. (Las propiedades que funcionan mejor en el entorno se volverán más frecuentes).

Este mecanismo puede explicar cómo y por qué la frecuencia relativa de las entidades con diferentes propiedades cambia con el tiempo. Los símbolos abstractos deben considerarse como marcadores de posición para fenómenos más concretos (como individuos, organizaciones, estrategias organizativas, etc.). (Hedström \& Bearman: 2009: 7-8)

Las entidades (por ejemplo, humanos, organizaciones, instituciones, etc.) de una determinada población, exhiben diferentes propiedades (por ejemplo, altura, peso, rasgos de personalidad, etc., para las personas; formas de organización, estructuras de decisión, protocolos de selección de miembros, normas, presupuestos, restricciones, etc., para entidades supraindividuales) cuya posesión beneficia en cierto ambiente. Las entidades que tengan ciertas propiedades en cierta magnitud (siempre cuantificable) tendrán una mayor frecuencia relativa en la población de un entorno determinado. Esto quiere decir que hay una correlación entre la posesión de esa propiedad/beneficio en una cierta magnitud y la frecuencia de los organismos que poseen dicha propiedad en dicha magnitud en una población y ambiente dado. El siguiente ejemplo ilustra la lógica general:

"En particular, la supervivencia diferencial de organismos basada en sus patrones conductuales puede conducir a una conducta óptima (óptima para la reproducción) en la población incluso en ausencia de toda elección o intención optimizadoras. Supongamos que el $10 \%$ de los organismos en una población de 100 integrantes procuran su alimento con tanta eficacia que dejan de promedio 10 descendientes que sobreviven hasta la adultez, mientras que el $90 \%$ restante deja sólo 5 . Si el comportamiento de los padres es transmitido (por 
REVCOM | ISSN 2451-7836 | Año 4, \#8 | mayo de 2019 | Contribuciones | e011

cualquier mecanismo) a la descendencia, la siguiente generación de organismos adultos incluirá una fracción de 100/550=2/11 18\% que muestra la conducta más eficiente. En el curso de unas pocas generaciones más, virtualmente todos los organismos mostrarán esa conducta. Si preguntamos por qué se muestra universalmente, la respuesta es que tiene mejores consecuencias. Este mecanismo opera a través de las generaciones." (Elster: 2007: 274).

Según Elster (2007: 287-298), una manera en que el mecanismo biológico de la selección natural puede influir en la conducta es mediante la selección de características físicas que inducen, por ejemplo, emociones que, a su vez, tienen impacto en las acciones. Este autor discute también otras explicaciones seleccionistas, naturalistas o no, mostrándose, en general, bastante escéptico. Pero sin descartar la posibilidad de ensamblar algunas realmente efectivas (como la ya citada de las tendencias a la acción de algunas emociones modeladas, quizá, por el mecanismo de replicación diferencial).

\section{Influencia social}

Para ejemplificar la utilización de modelos estocásticos de decisiones colectivas que incorporan la influencia social, nos referimos al trabajo de Matthew Salganik, Peter Sheridan Dodds y Duncan Watts, quienes intentaron cuantificar la desigualdad y la predictibilidad de resultados en la descarga de un conjunto de canciones. Crearon un mercado cultural web con fines experimentales, compuesto por 14,341 participantes reclutados de http://www.bolt.com, un sitio popular para adolescentes y jóvenes adultos. Los experimentos tuvieron lugar entre Octubre de 2004 y Marzo de 2005, con sujetos participantes de distintas nacionalidades, principalmente de EEUU. (ver estadística descriptiva de los sujetos en Salganik, Dodds \& Watts: 2006).

El estudio busca explorar algunos aspectos de la influencia social, intentando avanzar en la explicación de dos cuestiones características de los mercados culturales. Por un lado, la desigualdad. Es decir, la gran diferencia existente, en órdenes de magnitud, entre los productos culturales que resultan más difundidos y aquellos que tienen una difusión promedio (por ejemplo, Harry Potter en comparación con la casi totalidad de lo publicado, en el caso del mercado literario). Por otro lado, la impredictibilidad: la imposibilidad de predecir o anticipar cuáles productos tendrán más éxito (un hecho señalado tanto por los patrones de la industria cultural como por la investigación académica; en concreto, productos que se consideran 
REVCOM | ISSN 2451-7836 | Año 4, \#8 | mayo de 2019 | Contribuciones | e011

excelentes tienen un pobre desempeño de ventas, mientras que otros rechazados sistemáticamente por la industria se convierten en sensacionales éxitos de venta imprevistos). En el caso de los experimentos que comentamos, las medidas de éxito son función de la cantidad de descargas realizadas para las canciones ofrecidas.

Las interfaces diseñadas permitían a los sujetos experimentales acceder segmentadamente sólo a cierto tipo de información. Los participantes fueron asignados al azar a dos condiciones distintas. En una de ellas, la condición independiente, sólo disponían de los nombres de las bandas y sus canciones. En la otra, la condición de influencia social, podían observar además el número de descargas previas asociado a cada canción. Los autores refieren a la compleja problemática de la influencia social, haciendo referencia a investigaciones previas y poniendo énfasis en los procesos de imitación. En el caso que nos ocupa, la influencia social se limita a la posibilidad de imitar la conducta de otros participantes al acceder a la información sobre las descargas previas. Ambos experimentos empezaban con cero descargas. En el experimento 1, configurado en la condición de influencia social, las canciones y su número de descargas previas se presentaban en tres columnas ordenadas aleatoriamente para cada participante. En la condición independiente, la presentación de las canciones era la misma, pero sin ninguna información sobre descargas previas. En el experimento 2, en la condición de influencia social se presentaron las canciones en una única columna en orden descendente según el número de descargas. En la condición independiente, también fueron presentadas en una única columna, pero sin los conteos de descargas y en orden aleatorio para cada participante. A cada sujeto se le mostró una lista de 48 canciones desconocidas de bandas desconocidas. Mientras los participantes escuchaban una canción, se les pidió que le asignaran una calificación desde una a cinco estrellas, donde una estrella significaba "no me gusta" y cinco estrellas "me encanta". Luego se les dio la oportunidad de efectuar la descarga.

Para entender mejor la impredictibilidad, los participantes en la condición de influencia social fueron asignados al azar en ocho grupos distintos, cada uno de los cuales compartían la misma información, grupos que los autores denominan mundos de influencia social. Cada sujeto tenía información sólo sobre el comportamiento de los otros en su mundo de influencia. El objetivo fue observar en qué medida dos grupos indistinguibles de sujetos, con la misma condición inicial, y eligiendo del mismo conjunto de canciones y bandas pueden generar resultados diversos en el número de descargas de cada canción. 
REVCOM | ISSN 2451-7836 | Año 4, \#8 | mayo de 2019 | Contribuciones | e011

Para medir el éxito de cada canción se utilizó la cuota de mercado definida como:

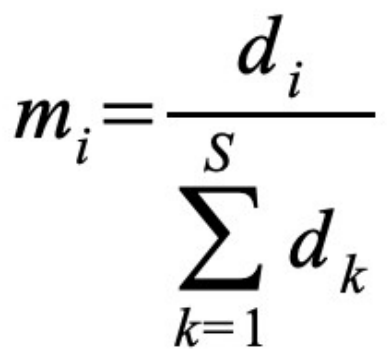

Siendo $d_{i}$ el número de descargas por canción $i$, $S$ el número de canciones y $\sum_{k=1}^{S} d_{k}$ el total de descargas. Esta definición del éxito está basada en el comportamiento de los sujetos, más que en su gusto sobre las canciones, medido por la calificación otorgada de 1 a 5 estrellas. Sin embargo, al comparar las dos medidas los autores observan que la probabilidad de descarga para una canción aumenta correlativamente al aumentar el promedio de sus calificaciones.

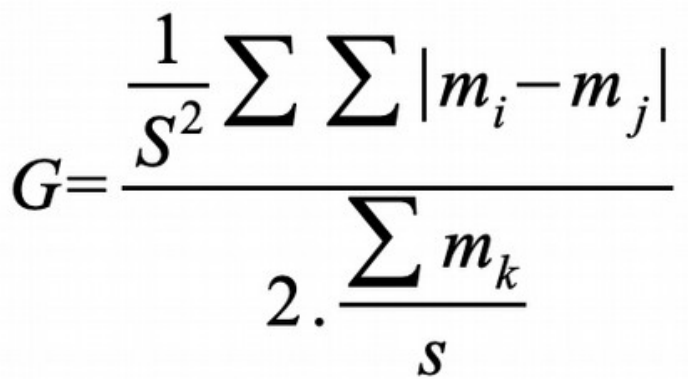

Para medir la desigualdad del éxito entre canciones, se utilizó el Coeficiente de Gini, que representa la diferencia esperada en la cuota de mercado entre dos canciones al azar. El Coeficiente puede caer entre los valores 0 y 1 , donde 0 representa completa igualdad y 1 completa desigualdad.

Así, en ambos experimentos, los ocho mundos de influencia social exhibieron mayor desigualdad, lo cual significa que las canciones populares fueron más populares y las impopulares menos populares, que en los mundos donde los individuos realizaron sus decisiones sin información sobre la conducta de otros. Además, la desigualdad de los resultados se incrementa cuanto más fuerte es la influencia social a la que son sujetos los individuos. 
REVCOM | ISSN 2451-7836 | Año 4, \#8 | mayo de 2019 | Contribuciones | e011

Para medir la imprevisibilidad se examinó cómo variaba el éxito de una canción a través de los mundos. Si una canción tuvo el mismo resultado en todos los mundos la imprevisibilidad es igual a 0 . Pero, si los resultados variaban a través de los diferentes mundos, había una imprevisibilidad inherente al éxito de la canción. Así, la impredictibilidad de una canción $i$ es la diferencia promedio en la cuota de mercado a través de todos los pares de mundos posibles:

$$
u_{i}=\frac{\sum \sum\left|m_{i, j}-m_{i, k}\right|}{(w)}
$$

Donde $m_{i, j}$ es la cuota de mercado de la canción $i$ en el mundo $j$, y es $(w)$ la cantidad de pares de mundos. La impredictibilidad $U$, para una condición experimental es entonces el promedio de la impredictibilidad de las canciones en esa condición.

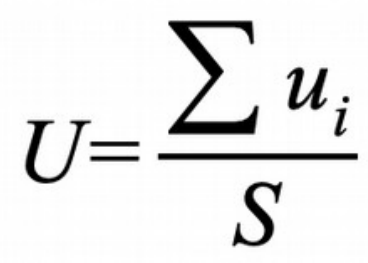

Los resultados demuestran que, en cada experimento, la impredictibilidad para una canción es mayor entre los mundos de influencia social que entre los mundos donde los individuos eligen independientemente. La impredictibilidad se da en distintos grupos de individuos, con características indistinguibles y respecto al mismo conjunto de canciones, por lo que, resulta inherente al proceso y no puede eliminarse conociendo más a las canciones o a los participantes.

Las pruebas de significación estadística para la desigualdad y la impredictibilidad entre la condición independiente y la condición de influencia social en ambos experimentos, resultaron, según los autores, estadísticamente significativas.

Salganik, Dodds y Watts (2006) reconocen la distancia que separa al experimento del mundo "real", considerando que la influencia social puede ser aún mayor en éste último, debido 


\section{VREVCOm \\ REVISTA CIENTIFICA DE LA REDCOM}

REVCOM | ISSN 2451-7836 | Año 4, \#8 | mayo de 2019 | Contribuciones | e011

a ciertos requerimientos que incrementaron el control sobre los sujetos participantes. A pesar de que estas diferencias limitan la relevancia del experimento, se considera que los resultados obtenidos permiten conjeturar que la influencia social ejerce un importante e inesperado efecto sobre los mercados culturales, generando conductas colectivas que "remiten" a lo que hacen otros a la hora de realizar elecciones sobre determinados productos culturales.

\section{Conclusión}

La sociología ha difundido, a veces de forma convencida y vigorosa, diversos tipos de analogías buscando esclarecer la vida social humana: física social, organismos sociales, sistemas y estructuras sociales, ecosistemas sociales. La analogía de los mecanismos cuenta al presente con un número creciente de adeptos y defensores entusiastas, aunque no tantos en el medio académico argentino. Ha sido nuestra intención, sin pretensiones de originalidad, presentar en nuestro medio la idea general y alguna de sus realizaciones, y deslizar, como lo haremos a continuación, algunas inquietudes epistemológicas que nos dejan estas exploraciones tentativas.

Cabe anotar, como primera cuestión polémica que emerge al inspeccionar sumariamente la literatura, la falta de consenso sobre la misma idea de mecanismo. Ello no obsta para que los investigadores no reconozcan la validez de aplicaciones empíricas que, si bien reivindican conceptos distintos, respetan un canon metodológico similar. Así, por ejemplo, herramientas afinadas por individualistas estrictos como Elster, para el caso de las emociones y la conducta, pueden ser reivindicadas por otros investigadores que reconocen, como el caso de Hedström, la necesidad de postular estructuras sociales como forma de obtener profundidad causal.

En este sentido, la discusión con respecto a los presupuestos metodológicos, como el individualismo, nos remite a la argumentación de las restricciones que solicitarían unas ciencias sociales con pretensión de entroncar con el marco de la concepción científica materialista moderna, y sobre la estructuración lógica de algunos conceptos invocados como el de causalidad. La fuerza de los argumentos individualistas proviene de, entre otras cosas, premisas metafísicas como la estructura temporal de la relación causal y el tipo de entidades que se postulan, o lógicas como la representación formal apropiada de las explicaciones, o incluso metodológicas como las que hemos mencionado más arriba. Además de estos problemas clásicos, se agregan las polémicas que en cantidad y en curso existen en filosofía de la mente y que se importan ipso facto al campo de las ciencias sociales al invocar la relación 


\section{REVCOM \\ REVISTA CIENTIFICA DE LA REDCOM}

REVCOM | ISSN 2451-7836 | Año 4, \#8 | mayo de 2019 | Contribuciones | e011

de superveniencia para dar cuenta de las relaciones Micro-Macro.

También en la dirección de la ubicación de las ciencias sociales en el cuadro general del conocimiento científico está el problema ontológico. ¿De qué cosas hablan exactamente las ciencias sociales? Ya hemos mencionado, más arriba, la idea de reducción en términos psicológicos y, en última instancia, biológicos. Dan Sperber $(1996,2011)$ por su parte, ha propuesto una ontología, para la explicación mecanística, que enmarca en una idea de las ciencias sociales como epidemiología de las representaciones, y que viene asociada a intentos paralelos de dar cuenta de los fenómenos de replicación cultural, cuestión que también hemos tratado brevemente aquí a propósito de los estudios de Salganik, Dodds y Watts.

Jon Elster $(2007,2012)$ ha advertido repetidamente, en tiempos recientes, contra las excesivas ambiciones en las ciencias sociales, y acusado de oscurantistas, hard o soft, a famosos teóricos (por ejemplo, a Marx, Bourdieu, Lévi-Strauss y Lacan, entre otros). El oscurantismo hard es modélico y cuantitativo; el soft es más literario. Los oscurantismos pueden producir daño o desperdicio. Generaciones de estudiantes repitiendo ad verecundiam ( y ad nauseam) teorías erróneas es desperdicio. La teoría del autismo de Bruno Bettelheim, que aparentemente culpabiliza a los padres, es un ejemplo de daño producido por oscurantismo soft. La caída de Long Term Capital Management con sus modelos de riesgo financiero sería un ejemplo de daño producido por oscurantismo cuantitativo hard. (Taleb: 2007) ha argumentado que el uso de algunos modelos financieros podría estar en los orígenes de muchas crisis, al generar exposiciones inducidas por computación errónea de eventos de muy baja probabilidad, pero altísimo impacto negativo). En particular, Elster se ha manifestado escéptico respecto de la relevancia empírica, en versiones sofisticadas, de muchos modelos al estilo de los aquí tratados (modelos basados en agentes, por ejemplo), pero señala la utilidad de algunos por su sencillez y transparencia lógica, citando los de Schelling como los descriptos sumariamente en el presente trabajo.

Siguiendo un precepto de Lakatos nos abstendremos de lanzar invectivas contra un programa de investigación en etapa de crecimiento. La voluntad de académicos convencidos sostendrá el embrión. El dictamen del tiempo, en la forma de desafíos lógicos y empíricos superados, decidirá. 
REVCOM | ISSN 2451-7836 | Año 4, \#8 | mayo de 2019 | Contribuciones | e011

\section{BIBLIOGRAFIA}

Bunge, M. (2007). A la caza de la realidad: la controversia sobre el realismo. Barcelona: Ed. Gedisa.

Elster, J. (1999). "A plea for Mechanisms" en Alchemies of the Mind, Cambridge UP, Cambridge UK.

Elster, J. (2007). Explaining Social Behavior, Cambridge UP, NY.

Elster, J. (2009). "Emotions" en Hedström y Bearman (2009).

Elster, J. (2012). "Hard and Soft Obscurantism in the Humanities and Social Sciences", Diogenes. Vol. 58(1-2), pp. 159-170.

Granovetter, M (1978). "Threshold Models of Collective Behavior". American Journal of Sociology, Vol.83, $\mathrm{N}^{\circ} 6$, pp. $1420-1443$

Harford, T. (2008). La lógica oculta de la vida. Buenos Aires: Ed. Temas de hoy.

Hedström, P \& Bearman, P. (2009). "What is Analytical Sociology All About? An Introductory Essay" en Hedström, P \& Bearman, P The Oxford Handbook of Analytical Sociology. (pp. 3-24) Oxford: Oxford UP.

Hofstadter, D. \& Sander, E. (2013): Surfaces and Essences. New York: Basic Books.

Kim, J. (2011). Philosophye of Mind. Boulder CO-USA, Westview Press

Kuhn, T.S. (1996): The Structure of Scientific Revolutions, 3rd Ed. Chicago; Chicago UP.

Lakoff, G. \& Johnson, M. (1980): Methaphors We Live By. Chicago: Chicago UP.

Machamer, P; Darden, L y Craver, F (2000). "Thinking about Mechanisms". Philosophy of Science, Vol.67, $\mathrm{N}^{\circ} 1$, pp. $1-25$

Salganik. M, Dodds. P \& Watts. D. (2006). "Experimental Study of Inequality and Unpredictability in an Artificial Cultural Market”. Science. Vol. 311, pp. 854-856

Shelling. C. T. (1971). "Dynamic Models of Segregatión”. Journal of Mathematical Sociology. Vol 1, pp 143-186. Sociology. Cambridge University Press.

Sperber, D. (1996). Explaining Culture: A Naturalistic Approach. Oxford: Blackwell.

Sperber, D. (2011). "A naturalistic ontology for mechanistic explanation in social sciences," en P. Demeulenaere (ed.) Analytical Sociology and Social Mechanisms, Cambridge University Press.

Taleb, N. N. (2007): The Black Swann; Random House, N.Y.

Watts, D. (2013) "Computational Social Science: exciting progress and future directions". The Bridge. Vol 43 , 14, pp. 5-10.

Westfall, R. (1980): La construcción de la ciencia moderna. Mecanismos y mecánica. Barcelona; Ed Labor. 